\title{
Incidencia Universitaria en las Actitudes hacia la Investigación: Adaptación de un Instrumento Breve
}

\author{
University Incidence on Attitudes towards Research: Adaptation of a Brief \\ Instrument
}

\author{
Juan Aníbal González-Rivera ${ }^{*}$ * Adam Rosario-Rodríguez ${ }^{2}$, Natasha Torres-Rivera ${ }^{3}$, Taimara Ortiz-Santiago ${ }^{4}$, \\ Vicmarie Sepúlveda-López ${ }^{\text {, }}$ Miriam Tirado de Alba ${ }^{6}$, \& Chardlyn M. González-Malavé 7 \\ 1 Ponce Health Sciences University, Ponce, Puerto Rico. (D) https://orcid.org/0000-0003-0622-8308 \\ 2 Carlos Albizu University, San Juan, Puerto Rico. (D) https://orcid.org/0000-0003-1603-5040 \\ 3 Carlos Albizu University, San Juan, Puerto Rico. (D) https://orcid.org/0000-0003-3975-4208 \\ 4 Carlos Albizu University, San Juan, Puerto Rico. (D) https://orcid.org/0000-0002-3944-4835 \\ 5 Carlos Albizu University, San Juan, Puerto Rico. (D) https://orcid.org/0000-0003-2426-4708 \\ 6 Carlos Albizu University, San Juan, Puerto Rico. (D) https://orcid.org/0000-0003-3898-3041 \\ 7 Carlos Albizu University, San Juan, Puerto Rico. (D) https://orcid.org/0000-0002-4286-6043 \\ * Correspondencia: jagonzalez@psm.edu.
}

Recibido: 1 junio 2021 | Aceptado: 29 julio 2021 | Publicado: 1 septiembre 2021

WWW.REVISTACARIBENADEPSICOLOGIA.COM

\section{Citar como:}

González-Rivera, J. A., Rosario-Rodríguez, A., Torres-Rivera, N., Ortiz-Santiago, T., Sepúlveda-López, V., Tirado de Alba, M., \& González-Malavé, C. M. (2021). Incidencia universitaria en las actitudes hacia la investigación: Adaptación de un instrumento breve. Revista Caribeña de Psicología, 5, e5809.

https://doi.org/10.37226/rcp.v5i1.5809

\section{RESUMEN}

El estudio tiene como objetivo adaptar un instrumento breve que mida incidencia universitaria en las actitudes de los estudiantes graduados hacia la investigación. Este proyecto es la fase inicial de una investigación mayor que pretende examinar la autoeficacia para investigar y su relación con otras variables académicas en una muestra de estudiantes graduados de Puerto Rico. Como base para la versión breve se utilizaron dos factores del Índice de Actitud hacia la Investigación. Participaron 308 estudiantes universitarios activos en escuela graduada (maestría y doctorado). El análisis factorial exploratorio reflejó una solución de dos factores que explicaban el 66.38\% de la varianza. Los factores fueron Incidencia de Profesores (5 ítems) e Incidencia Institucional (5 ítems). Para el factor 1, Incidencia del profesor, los índices de discriminación de los ítems fluctuaron entre .52 a .76 , obteniendo un alfa de Cronbach de .84. Para el Factor 2, Incidencia institucional, la discriminación de los ítems osciló entre .62 y .80, logrando un alfa de Cronbach de .88. Estos resultados sugieren que la escala tiene el potencial para medir este constructo en Puerto Rico y se recomienda su uso en futuras investigaciones.

Palabras Claves: actitudes, investigación, confiabilidad, propiedades psicométricas, validación

\section{ABSTRACT}

The study's objective is to adapt a brief instrument that measures university incidence in the attitudes of graduate students towards research. This project is the initial phase of other investigation that aims to examine self-efficacy 
to investigate and its relationship with other academic variables in a sample of graduate students from Puerto Rico. As a basis for the short version, two factors from the Attitude towards Research Index were used. Three hundred eight university students in graduate school (master's and doctorate) participated. The exploratory factor analysis reflected a solution of two factors that explained $66.38 \%$ of the variance. The factors were Professor Incidence (5 items) and Institutional Incidence (5 items). For factor 1, Professor Incidence, the discrimination indices of the items fluctuated between .52 to .76 , obtaining a Cronbach's alpha of .84 . For Factor 2, Institutional Incidence, the item discrimination ranged from .62 to .80 , achieving a Cronbach's alpha of .88 . These results suggest that the scale can measure this construct in Puerto Rico, and its use is recommended in future research.

Keywords: attitudes, research, reliability, psychometric properties, validation

\section{INTRODUCCIÓN}

El ejercicio de la investigación universitaria es un factor determinante en el proceso de adquisición de nuevos conocimientos (Mamani, 2011) y fundamental en el desarrollo de profesionales de alto calibre. De hecho, la actitud de los estudiantes hacia la investigación es un indicador sobre la calidad de la educación recibida dado que enseñar a investigar es un elemento transversal en la formación universitaria (Rojas et al., 2012). Se parte de la premisa que, a mejor actitud hacia la investigación, mayor formación científica y, en consecuencia, mayor éxito profesional.

A pesar que las actitudes de las personas hacia "algo" pueden modificarse o transformarse con el tiempo, tienen indubitablemente un elemento predictivo que nos permitirá inferir futuros comportamientos o patrones conductuales (Arellano et al., 2019; Kennedy et al., 2016; Ortega et al., 2018; QuezadaBerumen et al., 2019). Sin embargo, las actitudes hacia un objeto o una situación determinada -en este caso la investigación-no dependen exclusivamente de elementos motivacionales intrínsecos. En el contexto universitario, existen factores externos como, el estilo del docente, recursos disponibles, experiencia de la facultad y apoyo de la institución, que afectan las actitudes de los estudiantes hacia los procesos de investigación.

En este sentido, aquí la importancia de medir las actitudes de los estudiantes graduados hacia la investigación desde la incidencia percibida de sus profesores e instituciones universitarias. Uno de los instrumentos que existen para medir este constructo es el Índice de Actitud hacia la Investigación en Estudiantes (IAI) desarrollado en Colombia por Rojas et al. (2012). El instrumento original cuenta con 17 ítems distribuidos en tres factores: autoevaluación, inciden- cia de los profesores en las actitudes de los estudiantes e incidencia de la institución en este tipo de actitudes. A pesar que los autores no reportaron evidencias de validez, el instrumento obtuvo un índice de confiabilidad aceptable $(\alpha=.88)$.

El propósito del presente estudio es adaptar un instrumento breve que mida incidencia universitaria en las actitudes de los estudiantes graduados hacia la investigación. Para esto, se utilizaron dos de los tres factores del IAI: incidencia de los profesores (calidad de la formación y la enseñanza) e incidencia de la institución (contexto institucional). Esta nueva versión adaptada nos permitirá examinar concretamente el impacto que tiene la universidad en las actitudes de los estudiantes hacia la investigación; es decir, dos elementos extrínsecos de dicha actitud. En fin, se examinó la validez de constructo, la capacidad de discriminación de los ítems y la confiabilidad del instrumento.

\section{MÉTODO}

\section{Diseño de Investigación}

Se utilizó un diseño de tipo instrumental (Ato et al., 2013). El estudio fue aprobado por el Comité para la Ética en la Investigación de la Universidad Carlos Albizu en San Juan, Puerto Rico. El recogido de datos se realizó de forma electrónica y anónima mediante la plataforma PsychData.

\section{Participantes}

La muestra de esta investigación estuvo compuesta por 308 estudiantes universitarios activos en escuela graduada con edades que fluctuaron entre los 21 hasta los $40(M=29.34, D E=5.03)$. La mayoría de los participantes fueron del género femenino $(77.6 \%$, $n=239)$, con una preparación académica de grado de Maestría $(64.9 \%, n=200)$, que se encontraban en su 
primer $(27.6 \%, n=85)$ y segundo año de estudios graduados $(27.6 \%, n=85)$, con un aprovechamiento académico reportado entre 3.50 a $4.00(86.7 \%, n=267)$ en una escala de 1.00 a 4.00. La Tabla 1 muestra los datos sociodemográficos de los participantes en su totalidad.

La mayoría de los participantes indicaron que sus programas académicos requieren completar una investigación ya sea de tesis o disertación $(71.8 \%, n=$ 221). Poco más de la mitad de los estudiantes $(53.9 \%$, $n=166$ ) no han tenido la oportunidad de colaborar en el desarrollo de alguna investigación en su Universidad al momento de este estudio. La mayoría (69.5\%, $n=214$ ) indicó que no está participando en algún proyecto de investigación en su Universidad. Sin embargo, a gran parte de los estudiantes de la muestra $(77.6 \%, n=239)$ indicaron que les interesa la investigación.

Tabla 1

Datos Sociodemográficos.

\begin{tabular}{lcc}
\hline & $n$ & $\%$ \\
\hline Sexo & 239 & 77.6 \\
$\quad$ Femenino & 68 & 22.1 \\
$\quad$ Masculino & 1 & 0.3 \\
Otro & & \\
Preparación Académica & 200 & 64.9 \\
$\quad$ Maestría & 105 & 34.1 \\
$\quad$ Doctorado & 3 & 1.0 \\
Post Doctorado & & \\
Año Académico & 85 & 27.6 \\
$\quad$ Primero & 85 & 27.6 \\
Segundo & 53 & 17.2 \\
Tercero & 28 & 9.1 \\
Cuarto & 57 & 18.5 \\
Cinco o más & & \\
Promedio Académico Acumulado & 267 & 86.7 \\
3.50 - 4.00 & 35 & 11.4 \\
$3.00-3.49$ & 4 & 1.3 \\
$2.50-2.99$ & 2 & 0.6 \\
2.00 - 2.49 & 0 & 0.0 \\
Menos de 1.99 & & \\
\hline
\end{tabular}

\section{Instrumentos}

Índice de Actitud hacia la Investigación en Estudiantes (IAI). El instrumento original fue desarrollado por Rojas et al. (2012); el mismo es una medida global de la actitud estudiantil hacia la investigación bajo un modelo de tres dimensiones que establecen la predisposición hacia la investigación: el contexto institucional, la calidad de la formación y las motivaciones intrínsecas del estudiante. No obstante, basado en los intereses investigativos de los autores del presente estudio, se utilizaron solo dos dimensiones del instrumento: incidencia de los profesores e incidencia de la institución, incluyendo el ítem 5 del factor de Autoevaluación. La versión administrada, la cual denominaremos desde ahora Índice de Actitud hacia la Investigación en Estudiantes versión adaptada (IAI-A), consta de 12 ítems con una escala de respuesta tipo Likert de cinco puntos que va desde Nunca (0) hasta Siempre (4).

\section{Análisis de Datos}

Para analizar los datos se utilizó el programa estadístico IBM SPSS Statistics (versión 26). Se realizó un análisis de factores exploratorio (AFE), análisis de discriminación para los ítems, análisis de consistencia interna mediante el coeficiente alfa de Cronbach y análisis de correlación Pearson. Para el AFE se utilizó una estimación de máxima verosimilitud, una extracción Principal Axis Factoring, una rotación oblicua y para la selección de los factores se utilizó el criterio de valores Eigen que fueran igual o mayor de 1.00. Para mantener la robustez en el análisis, todo ítem que tuviera una carga factorial menor de .50 (Stevens, 2002). El índice de discriminación se calculó con la correlación ítem total $\left(r_{b i s}\right)$, el cual debe ser mayor a .30 (Kline, 2005). La confiabilidad se calculó con el coeficiente alfa de Cronbach. Field (2013) indica que índices mayores a .70 son aceptables.

\section{RESULTADOS}

\section{Análisis de Factores Exploratorio}

El AFE demostró buenos valores en la adecuacidad del tamaño de la muestra con relación a la cantidad de ítems en los índices de Kaiser-Meyer-Olkin y la esfericidad de Bartlett $\left(K M O=.93, \chi^{2}(66)=1,935.36\right.$, $p<.001)$. Se detectaron dos factores que explican el $60.88 \%$ de la varianza (Factor $1=51.17 \%$; Factor $2=$ 9.71\%). En este análisis los ítems 9 y 12 no lograron cargas factoriales satisfactorias por lo que se eliminaron y se volvió a realizar el análisis sin estos ítems.

Al realizar el segundo análisis de factores exploratorio la adecuacidad del tamaño de la muestra se mantuvo $\left(K M O=.92, \chi^{2}(45)=1,713.21, p<.001\right)$. Se mantuvo una estructura de dos factores explicando 
un $66.38 \%$ de la varianza, sin tener que eliminar ítems en esta ocasión. Finalmente, los ítems que se agruparon en el Factor 1 fueron la mayoría ítems que comprendían el factor de Incidencia de Profesores con la excepción de que en este factor también se agrupó el ítem 16 que originalmente pertenecía al factor de Incidencia Institucional. Por otro lado, los ítems que se agruparon para el Factor 2 incluyen en su mayoría ítems originales del factor de Incidencia Institucional incluyendo, además, al ítem 5 que originalmente pertenecía al factor de Autoevaluación. De esta manera la escala quedó con dos factores con cinco ítems por cada factor. La Tabla 2 muestra los ítems y su agrupación factorial con sus cargas factoriales.

Tabla 2

Cargas factoriales de los ítems.

\begin{tabular}{|c|c|c|}
\hline Ítems & $\begin{array}{l}\text { Factor } 1 \\
\text { IP }\end{array}$ & $\begin{array}{c}\text { Factor } 2 \\
\text { IINT }\end{array}$ \\
\hline $\begin{array}{l}\text {-Los profesores de mi carrera me aseso- } \\
\text { ran bien para realizar investigaciones. }\end{array}$ & .78 & \\
\hline $\begin{array}{l}\text {-Mis profesores están bien preparados en } \\
\text { investigación científica y tecnológica. }\end{array}$ & .71 & \\
\hline $\begin{array}{l}\text {-En mis cursos regulares me enseñan el } \\
\text { proceso de investigación científica. }\end{array}$ & .64 & \\
\hline $\begin{array}{l}\text {-Los profesores me exigen normas meto- } \\
\text { dológicas para la presentación de los tra- } \\
\text { bajos académicos. }\end{array}$ & .64 & \\
\hline $\begin{array}{l}\text {-Mis profesores confían en mis capacida- } \\
\text { des para realizar investigación científica. }\end{array}$ & .57 & \\
\hline $\begin{array}{l}\text {-En mi Universidad existen incentivos } \\
\text { académicos o económicos para que los es- } \\
\text { tudiantes realicen investigación científica. }\end{array}$ & & -.80 \\
\hline $\begin{array}{l}\text {-En mi Universidad se realizan convoca- } \\
\text { torias regulares para vincularse en pro- } \\
\text { yectos de investigación. }\end{array}$ & & -.71 \\
\hline $\begin{array}{l}\text {-En mi Universidad cuento con infraes- } \\
\text { tructura para la investigación científica. }\end{array}$ & & -.67 \\
\hline $\begin{array}{l}\text {-Mi Universidad se preocupa por actuali- } \\
\text { zar el conocimiento científico. }\end{array}$ & & -.63 \\
\hline $\begin{array}{l}\text {-Considero que en mi institución existe } \\
\text { un buen ambiente para la investigación. }\end{array}$ & & -.63 \\
\hline Varianza Explicada & $54.85 \%$ & $11.53 \%$ \\
\hline Valor Eigen & 5.49 & 1.15 \\
\hline Media & 14.05 & 9.82 \\
\hline Desviación Estándar & 4.74 & 5.71 \\
\hline
\end{tabular}

Nota. IP = Incidencia de Profesores; IINT = Incidencia Institución.

\section{Discriminación y Consistencia Interna}

Para los dos factores identificados en el AFE se examinaron los índices de discriminación de los ítems, así como la consistencia interna de los factores. Para el factor 1 de Incidencia de Profesores los índices de discriminación fluctuaron entre .52 a .76 obteniendo a su vez un alfa de Cronbach de .84. Para el Factor 2 de Incidencia Institucional la discriminación de los ítems fluctuó entre .62 a .80 logrando un alfa de Cronbach de 88 .

\section{Correlación entre Factores}

Se examinó la relación entre los factores (Incidencia de Profesores e Incidencia Institucional) mediante el análisis de correlación Pearson ya que al examinar la relación a través de un diagrama de dispersión se encontró que los factores tienen una relación lineal entre ellos. Ante esto, los resultados obtenidos de la correlación de Pearson demostraron que entre ambos factores existe una relación moderada alta (Champion, 1981) estadísticamente significativa $(r=.68, p<$ $.001)$.

Las propiedades psicométricas obtenidas demuestran que la versión adaptada del Índice de Actitud hacia la Investigación en Estudiantes (IAI-A) refleja indicadores adecuados de validez y consistencia interna. Se concluye que el IAI-A es un instrumento útil de medición para futuros estudios que deseen investigar la incidencia de los aspectos universitarios en la formación de los estudiantes graduados de Puerto Rico. Estudios de esta índole ayudarían a que las universidades analicen la forma en que están promoviendo las actividades investigativas en sus programas graduados y, además, conocer como el profesorado contribuye o incentiva en este proceso. El lenguaje sencillo y brevedad del IAI-A, favorece su utilización en cursos de metodología de la investigación a modo que los profesores puedan conocer la actitud de los estudiantes y así optimizar el proceso de enseñanza.

Futuros estudios deben explorar la validez concurrente del AIA-A mediante pruebas validadas que midan actitudes hacia la investigación. Además, se debe realizar un nuevo estudio para realizar un análisis factorial confirmatorio para examinar la estructura interna del IAI-A, y explorar la validez conver- 
gente y discriminante del mismo. En cuanto a las limitaciones del estudio, contamos con poca participación masculina y la selección de los participantes no fue aleatoria. Esto puede limitar la generalización de los resultados.

Financiamiento: La presente investigación no fue financiada por alguna entidad ni patrocinador.

Conflicto de Intereses: No existen conflictos de intereses de parte del autor de la investigación.

Aprobación de la Junta Institucional para la Protección de Seres Humanos en la Investigación: Comité para la Ética en la Investigación de la Universidad Carlos Albizu en San Juan, Puerto Rico.

Consentimiento o Asentimiento Informado: Todo participante completó un consentimiento informado.

Proceso de Revisión: Este estudio ha sido revisado por pares externos en modalidad de doble ciego.

\section{REFERENCIAS}

Ato, M., López, J. J., \& Benavente, A. (2013). Un sistema de clasificación de los diseños de investigación en psicología. Anales de Psicología, 29(3), 1038-1059.

Arellano, A., Gaeta, M. L.; Peralta, F., \& Cavazos, J. (2019). Actitudes hacia la discapacidad en una universidad mexicana. Revista Brasileira de Educação, 24, 1- 20. http://dx.doi.org/10.1590/s1413-24782019240023

Champion, D. J. (1981). Basic statistics for social research (2nd ed.).
McMillan.

Field, A. P. (2013). Discovering statistics using IBM SPSS Statistics ( 4 a . ed.). SAGE.

Kennedy, J. P., Quinn, F., \& Taylor, N. (2016). The school science attitude survey: A new instrument for measuring attitudes towards school science. International Journal of Research \& Method in Education, 39(4), 422-445. https://doi.org/10.1 080/1743727X.2016.1160046

Kline, T. J. (2005). Psychological testing: A practical approach to design and evaluation. Sage.

Mamani, O. J. (2011). Actitud hacia la investigación y su importancia en la elección de la modalidad de tesis para optar el título profesional. Revista Científica de Ciencias de la Salud, 4(4), 22 - 27. https://doi.org/10.17162/rccs.v4i1.82

Ortega, R. J., Veloso, R. D., \& Hansen, O. S. (2018). Percepción y actitudes hacia la investigación científica. Revista de Investigación en Ciencias Sociales y Humanidades, 5(2), 101-108. https://dx.doi.org/10.30545/academo.2018.jul-dic.2

Quezada-Berumen, L., Moral de la Rubia, J., \& LanderoHernández, R. (2019). Validación de la Escala de Actitud hacia la Investigación en estudiantes mexicanos de psicología. Revista Evaluar, 19(1), 1-16.

Rojas, H. M., Méndez, R., \& Rodríguez, A. (2012). Índice de actitud hacia la investigación en estudiantes del nivel de pregrado. Entramado, 8(2), 216-229.

Stevens, J. (2002). Applied multivariate statistics for the social sciences (4⿳亠口冋. ed.). Lawrence Erlbaum.

Obra bajo licencia de Creative Commons Atribución 4.0 Internacional (CC BY 4.0).

(C) 2021 Autores. 